\title{
Nomogram to predict the progression of patients with primary membranous nephropathy and nephrotic syndrome
}

\author{
Lili Liu $^{1} \cdot$ Haitao Wang ${ }^{1} \cdot$ Ban Zhao $^{1} \cdot$ Xin Liu $^{1} \cdot$ Ying Sun $^{1} \cdot$ Yonghui Mao $^{1} \mathbb{C}$
}

Received: 28 October 2020 / Accepted: 11 April 2021 / Published online: 28 April 2021

(c) The Author(s) 2021

\begin{abstract}
Background The outcome of patients with primary membranous nephropathy (pMN) who present with nephrotic syndrome (NS) is variable and difficult to predict. The goal of this study was to develop a nomogram to predict the risk of progression for specific individuals.

Methods This retrospective study involved biopsy-proven patients with pMN and NS treated between January 2012 and June 2018. The primary outcome of our investigation was progression, defined as a reduction of estimated glomerular filtration rate (eGFR) that was equal to or over $20 \%$ compared with baseline at the end of follow-up or the onset of end-stage renal disease (ESRD). We used backwards stepwise logistic regression analysis to create a nomogram to predict prognosis. The model was validated internally using bootstrap resampling.

Results A total of 111 patients were enrolled. After a median follow-up of 40.0 months (range 12-92 months), 18.9\% (21/111) patients showed progression. Backwards stepwise selection using the Akaike information criterion (AIC) identified the following four variables as independent risk factors for progression, which were all used in the nomogram: age $\geq 65$ years [odds ratio (OR) 7.004; 95\% confidence interval (CI) 1.783-27.505; $p=0.005$ ], Ln (sPLA2R-Ab) (OR 2.150; 95\% CI 1.293-3.577; $p=0.003)$, Ln (proteinuria) (OR 5.939; 95\% CI 1.055-33.436; $p=0.043)$ and $\mathrm{Ln}(\mathrm{U} \alpha 1 \mathrm{~m} / \mathrm{Cr})(\mathrm{OR} 2.808 ; 95 \%$ CI 1.035-7.619; $p=0.043$ ). The discriminative ability and calibration of the nomogram revealed good predictive ability, as indicated by a C-index of 0.888 (95\% CI $0.814-0.940)$ and a bootstrap-corrected C-index of 0.869 ; calibration curves were also well fitted. A receiver operating characteristic (ROC) curve for the nomogram score revealed significantly better discrimination than each of the three risk factors alone, including Ln (sPLA2R-Ab) [area under the curve (AUC) 0.769],

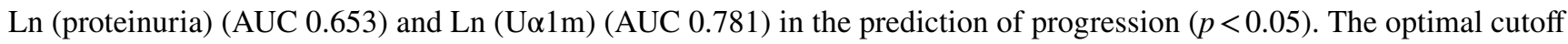
value of the nomogram score was 117.8 with a positive predictive value of $44.4 \%$ and a negative predictive value of $98.5 \%$. Conclusion The nomogram successfully achieved good predictive ability of progression for patients with pMN who present with NS. It can therefore help clinicians to individualize treatment plans and improve the outcome of pMN.
\end{abstract}

Keywords Primary membranous nephropathy $\cdot$ Nephrotic syndrome $\cdot$ Nomogram $\cdot$ Prognosis

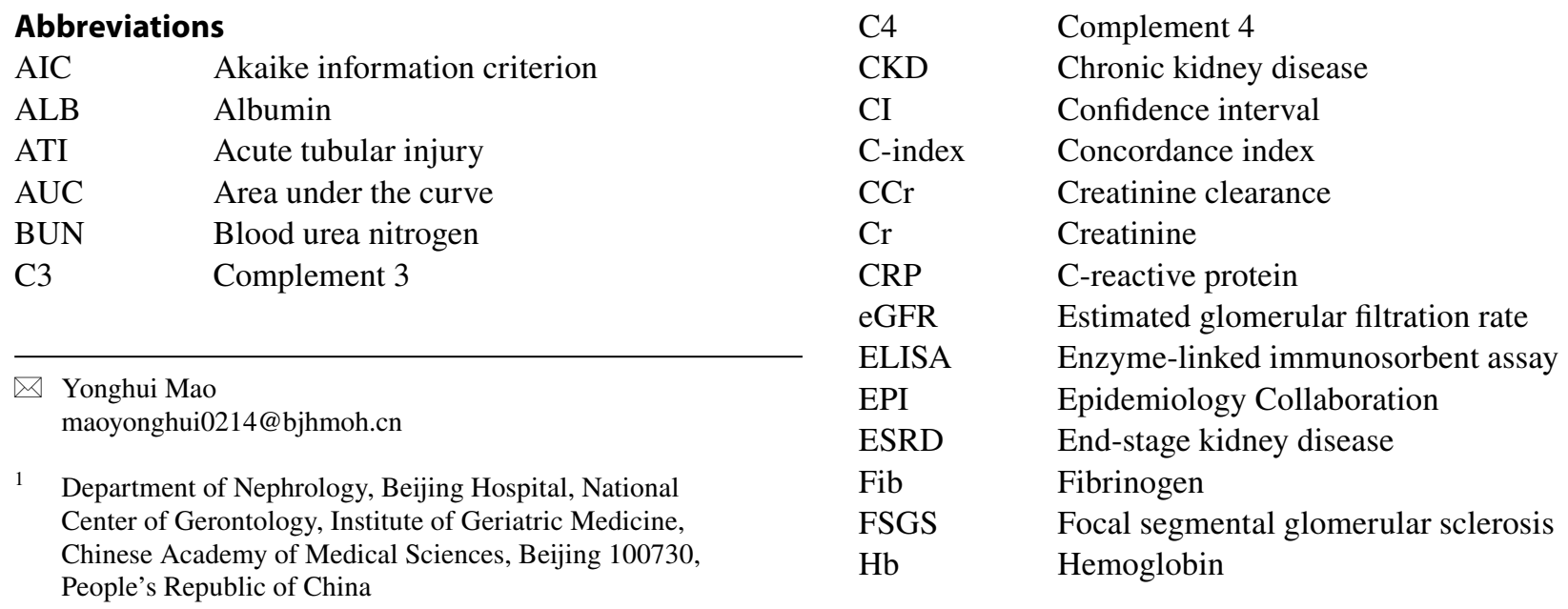




$\begin{array}{ll}\text { IF } & \text { Interstitial fibrosis KDIGO: Kidney Disease } \\ & \text { Improving Global Outcomes } \\ \text { LDL-C } & \text { Low-density lipoprotein cholesterol } \\ \text { LMW } & \text { Low molecular weight } \\ \text { HDL-C } & \text { High-density lipoprotein cholesterol } \\ \text { IgA } & \text { Immunoglobulin A } \\ \text { IgG } & \text { Immunoglobulin G } \\ \text { IgM } & \text { Immunoglobulin M } \\ \text { IQR } & \text { Interquartile range } \\ \text { Ln } & \text { Natural logarithm } \\ \text { NAG } & \text { N-Acetyl- } \beta \text {-D glucosaminidase } \\ \text { NR } & \text { Non-remission } \\ \text { NS } & \text { Nephrotic syndrome } \\ \text { PLT } & \text { Blood platelet count } \\ \text { pMN } & \text { Primary membranous nephropathy } \\ \text { PR } & \text { Partial remission } \\ \text { RBP } & \text { Retinol binding protein } \\ \text { ROC } & \text { Receiver operating characteristic } \\ \text { SCr } & \text { Serum creatinine } \\ \text { sPLA2R-Ab } & \text { Serum phospholipase A2 receptor antibody } \\ \text { TA } & \text { Tubular atrophy } \\ \text { TCHO } & \text { Total cholesterol } \\ \text { TG } & \text { Triglyceride } \\ \text { U } 1 \text { 1m } & \text { Urinary } \alpha 1 \text {-macroglobulin } \\ \text { UIgG } & \text { Urinary immunoglobulin G } \\ \text { UTf } & \text { Urinary transferrin } \\ \text { 24hUpro } & \text { 24-H proteinuria } \\ \text { VH } & \text { Vascular hyalinosis } \\ & \end{array}$

\section{Introduction}

Primary membranous nephropathy (pMN) is an autoimmune glomerular disease and one of the leading causes of nephrotic syndrome in adults. In China, the proportion of patients with pMN and primary glomerulopathy increased from $16.8 \%$ between 2003 and 2007 to $29.2 \%$ between 2008 and 2012 in all groups, and it increased from 53.21 to $70.23 \%$ in patients aged $\geq 60$ years [1]. $\mathrm{MN}$ is characterized by striking granular aggregations of immunoglobulin $\mathrm{G}(\mathrm{IgG})$ and electron-dense deposits along the subepithelial aspect of the glomerular basement membrane [2]. Recent breakthroughs indicate that M-type phospholipase A2 receptor (PLA2R), a transmembrane protein located on podocytes, acts as a major antigenic target in pMN. Studies have shown that serum PLA2R antibodies (sPLA2R-Abs) were present in $52-86 \%$ of patients with MN, with a specificity of $96-99 \%$ for $\mathrm{pMN}[3-7]$.

The clinical course and outcome of pMN are variable. Patients with subnephrotic proteinuria usually have excellent long-term renal survival. However, prognosis tends to vary among patients with proteinuria within the nephrotic range. Spontaneous remission of nephrotic syndrome occurs in approximately $20-25 \%$ of cases; however, approximately $30 \%$ of patients proceed to end-stage renal disease (ESRD) after 10 years [8]. Therefore, it is vital that we identify methods to determine risk factors for renal outcome for patients with pMN who present with NS. For many years, the recommended standard for treating such patients was to evaluate the extent of proteinuria; according to the 2012 Kidney Disease: Improving Global Outcomes (KDIGO) guidelines, patients with $>6$ months of proteinuria ( $>4 \mathrm{~g} /$ day) should receive immunosuppression [9]. However, this risk stratification lacks specificity, as a substantial proportion of such patients may still go into spontaneous remission [10]. Thus, there is an urgent need to identify sensitive and specific predictors that can be used alongside the evaluation of proteinuria. Previous studies demonstrated that the excretion of urinary IgG or low-molecular-weight (LMW) proteins including $\beta 2$-microglobulin ( $\beta 2 \mathrm{~m}), \alpha 1$-microglobulin $(\alpha 1 \mathrm{~m})$, and retinol binding protein (RBP) could predict the disease outcome of patients with pMN [11-14]. Two recent investigations suggested that sPLA2R-Ab is a useful biomarker for predicting prognosis and guiding treatment in $\mathrm{pMN}$ patients $[15,16]$. Higher levels of this antibody are associated with a lower chance of spontaneous or immunosuppressive therapy-induced remission and a higher risk of renal function deterioration.

Although several clinical parameters and biomarkers have been identified as predictors for $\mathrm{pMN}$, these data were derived from separate studies; there is currently no multivariate predictive model for pMN. In 1997, the Toronto Risk Score was proposed as a useful means of predicting renal outcome in patients with pMN and included three parameters: time-averaged proteinuria (highest sustained proteinuria over a 6-month period), creatinine clearance $(\mathrm{CCr})$ at diagnosis, and the slope of $\mathrm{CCr}$ over a 6-month period [17]. Nevertheless, this model was developed some time ago and did not consider the more recently identified predictors including SPLA2R-Ab and urinary biomarkers. Predictive models that include both traditional and new parameters may enable a much more informative assessment of outcome in patients with pMN.

We carried out this retrospective study using a cohort of patients with pMN who presented with NS. Our aim was to develop a novel prognostic nomogram and a prognostic score for renal outcome using a broad spectrum of clinical, laboratory, and pathological parameters available at baseline. We expect that this nomogram could be used to accurately and conveniently predict the progression of patients with pMN and thereby guide clinicians to optimize treatment strategy. 


\section{Materials and methods}

\section{Study population}

We conducted a retrospective study on a cohort of pMN patients who underwent native renal biopsy at Beijing Hospital between January 2012 and June 2018. The inclusion criteria were nephrotic syndrome (proteinuria $\geq 3.5 \mathrm{~g} /$ day and serum albumin $\leq 30 \mathrm{~g} / \mathrm{L}$ ) and an eGFR $\geq 30 \mathrm{~mL} /$ $\min / 1.73 \mathrm{~m}^{2}$. Our exclusion criteria were as follows: (1) patients with secondary MN, including autoimmune diseases (lupus nephritis and Sjögren syndrome), infectionrelated MN (hepatitis B virus-associated MN, hepatitis $C$ virus-associated $\mathrm{MN}$, human immunodeficiency virusassociated $\mathrm{MN}$, and syphilis, MN correlated to malignancies or exposure to toxic agents); (2) a follow-up duration of less than 1 year; (3) the use of immunosuppressive drugs within 3 months prior to kidney biopsy (Supplementary Fig. 1). Baseline and follow-up data were acquired from hospital medical records. Treatment options were in compliance with the KDIGO Guideline for glomerulonephritis.

All study procedures were conducted according to the 2008 Declaration of Helsinki and good clinical practice guidelines. The study was approved by the Ethics Committee of Beijing Hospital (Reference number: 2018BJYYEC-140-1).

\section{Data collection}

Baseline data were collected at the time of renal biopsy, including age, gender, history of hypertension, history of diabetes, hemoglobin ( $\mathrm{Hb}$ ), albumin (ALB), serum creatinine ( $\mathrm{SCr}$ ), blood urea nitrogen (BUN), uric acid (UA), total cholesterol (TCH), triglyceride, low-density lipoprotein cholesterol (LDL-C), high-density lipoprotein cholesterol (HDL-C), immunoglobulin G (IgG), 24-h proteinuria (24hUpro), urinary IgG corrected by creatinine (UIgG/Cr), urinary transferrin corrected by creatinine (UTf/Cr), urinary $\alpha 1$-microglobulin corrected by creatinine $(\mathrm{U} \alpha 1 \mathrm{~m} / \mathrm{Cr})$ and $N$-acetyl- $\beta$-D-glucosaminidase corrected by creatinine (NAG/Cr). Estimated glomerular filtration rate (eGFR in $\mathrm{mL} / \mathrm{min} / 1.73 \mathrm{~m}^{2}$ ) was calculated by the equation put forward by the Chronic Kidney Disease Epidemiology Collaboration (CKD-EPI) and was categorized according to the KDIGO 2012 Clinical Practice Guideline.

Serum was collected from each patient at the time of renal biopsy and stored at $-80{ }^{\circ} \mathrm{C}$. These samples were subsequently thawed to allow serum PLA2R antibody (sPLA2R-Ab) levels to be determined by a commercially available enzyme-linked immunosorbent assay (ELISA) kit (EUROIMMUN AG, Lubeck, Germany). In accordance with the manufacturer guidelines, sPLA2R-Ab levels $\geq 20$ $\mathrm{RU} / \mathrm{mL}$ were considered positive.

For all patients, kidney biopsy was performed at the time of diagnosis. Pathological kidney examination included light microscopy, immunofluorescence, and electron microscopy. Direct immunofluorescence analysis was performed on frozen sections to detect $\operatorname{IgG}, \operatorname{Ig} A, \operatorname{IgM}, \mathrm{C} 3, \mathrm{C} 4$, and C1q. Glomerular MN lesions were classified into four stages (I, II, III and IV) based on Ehrenreich and Churg's criteria. A number of characteristics were evaluated, including the presence or absence of focal segmental glomerular sclerosis (FSGS), acute tubular injury (ATI), and vascular hyalinosis $(\mathrm{VH})$. The degree of tubular atrophy (TA) and interstitial fibrosis (IF) were rated on a scale of 0,1 , or 2 based on the percentage of affected tubules or the extension of $\operatorname{IF}(<25$, $25-50,>50 \%$ ), respectively.

\section{Outcome}

Our primary outcome was progression. This was defined as a reduction of estimated glomerular filtration rate (eGFR) that was equal to or over $20 \%$ compared with baseline at the end of follow-up or the onset of ESRD; this definition was in accordance with the risk stratification set out by the 2019 KDIGO Controversies Conference Report [18]. Followup time was from the time of renal biopsy to one of three events: ESRD, loss to follow-up, or end of the study (31 December 2019).

\section{Statistical analysis}

Categorical variables are reported as whole numbers and proportions while continuous variables are reported as medians with interquartile ranges (IQRs). Clinical and laboratory variables that are associated with progression risk were assessed a priori based on clinical importance, scientific knowledge, and predictors that were identified in previously published articles. The associations of relevant variables with progression were assessed using a logistic regression model. Backwards stepwise selection using the Akaike information criterion (AIC) was used to identify variables to be incorporated into the multivariable logistic regression model. Odds ratios (ORs) were presented with their $95 \%$ confidence intervals (CIs). Selected variables were incorporated into the nomogram to predict the probability of progression using statistical software (rms in R, version 4.0.2; http://www.R-project.org).

Model accuracy was verified using two parameters: discrimination and calibration. The predictive performance and discrimination ability of the nomogram were measured using the concordance index (C-index). The $\mathrm{C}$-index estimates the probability of concordance between predicted and observed outcomes in rank order and is equivalent to the area under 
the receiver operating characteristic (ROC) curve. Generally, a $\mathrm{C}$-index $\geq 0.70$ is suggestive of a good fit. Calibration was evaluated using a calibration plot (a graphic representation of the relationship between the observed outcome frequencies and the predicted probabilities) with a bootstrapped sample of the study group. In a well-calibrated model, the predictions should fall on a $45^{\circ}$ diagonal line. Internal validation of the final model was performed with the 1000 bootstrap sample procedure to calculate a C-index that incorporated relative correction.

Finally, the nomogram was used to calculate the total scores for each patient. ROC curve analysis was used to identify the optimal cutoff value that was determined by maximizing the Youden index. The accuracy of the optimal cutoff value was assessed by sensitivity, specificity, and predictive values, and by likelihood ratio.

In all analyses, tests were two-sided, and $p<0.05$ was considered to indicate statistical significance. All analyses were performed using SPSS, version 25.0 (IBM Corp., Armonk, NY, USA) and R version 4.0.2 (Foundation for Statistical Computing, Vienna, Austria; http://www.R-proje ct.org).

\section{Results}

\section{Baseline clinical characteristics and outcome}

Baseline clinical characteristics and outcome data for the 111 patients enrolled on this study are given in Table 1 . The median patient age was 57 years (IQR 41-66 years), and $61.3 \%(68 / 111)$ of the patients were male. At the time of kidney biopsy, patients presented with a median eGFR of $99.2 \mathrm{~mL} / \mathrm{min} / 1.73 \mathrm{~m}^{2}$ and proteinuria of $5.7 \mathrm{~g} /$ day. Eightyone $(73.0 \%)$ patients were positive for SPLA2R-Ab as determined by ELISA, with a cutoff value of $20 \mathrm{RU} / \mathrm{mL}$; median level of sPLA2R-Ab was 212.6 RU/mL (IQR 75.4-416.2). Thirty patients were negative for sPLA2R-Ab with a median level of 5.1 U/mL (IQR 3.5-6.4). The levels of urinary $\mathrm{IgG} / \mathrm{Cr}, \mathrm{Tf} / \mathrm{Cr}, \alpha 1 \mathrm{~m} / \mathrm{Cr}$, and NAG/Cr were $14.7 \mathrm{mg} / \mathrm{g}$ (IQR 8.7-29.1), $2.2 \mathrm{mg} / \mathrm{g}$ (1.3-4.0), $307.5 \mathrm{mg} / \mathrm{mg}$ (IQR, 207.7-469.9), and 37.1 U/g (IQR 24.5-49.0), respectively.

Based on kidney biopsy, MN stages I, II, and III, were identified in $45.9 \%, 40.5 \%$, and $5.4 \%$ of patients, respectively. Obsolescent glomeruli were present in 2.1 (0-6.7) of glomeruli, $18.0 \%$ of patients (20/111) had FSGS, $48.65 \%$ of patients $(54 / 111)$ had $\mathrm{VH}$, and $20.7 \%$ of patients (23/111) had ATI. TA was graded as stage 0 in $93(83.8 \%)$ patients, stage 1 in $18(16.2 \%)$ patients and stage 2 in 0 patients $(0 \%)$, while IF was rated as stage 0,1 , and 2 , in 92 $(82.9 \%)$ patients, $19(17.1 \%)$ patients, and $0(0 \%)$ patients, respectively.
After a median follow-up of 40 months (range 12-92 months), $18.9 \%$ (21/111) of patients showed progression at the end of follow-up and remained within the nephrotic range of proteinuria. Compared with patients without progression, those with progression were significantly older and had significantly higher levels of sPLA2R-Ab and proteinuria and urinary biomarkers (UIgG/Cr, $\mathrm{U} \alpha 1 \mathrm{~m} / \mathrm{Cr}$, UTf/Cr, and NAG/Cr; all $p<0.05)$ but significantly lower eGFR and serum albumin levels $(p<0.05)$ (Supplementary Table 1). However, histological and immunofluorescence analyses revealed that there were no significant differences between the two groups with regard to MN stages, C3 deposits, or the presence of ATI. Furthermore, all of the patients with progression were shown to be positive for SPLA2R-Ab and $95.2 \%$ of these patients $(20 / 21)$ had received standard immunosuppressive treatment during follow-up.

\section{Model specifications and predictors of progression}

sPLA2R and urinary biomarker data were not normally distributed and therefore underwent natural logarithmic (Ln) transformation. Univariate logistic analysis identified that age $\geq 65$ years, albumin, CKD stage, Ln (proteinuria),

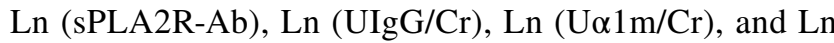
(NAG/Cr), were all significantly associated with progression $(p<0.05)$. These risk factors were then selected as candidate variables for the final prediction model. Backwards stepwise selection using the AIC and a logistic regression model identified the following four variables that had the strongest association with progression risk: age $\geq 65$ years, Ln (pro-

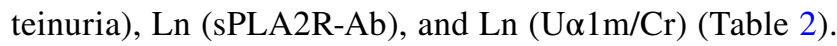
Multivariable analysis further identified that age $\geq 65$ years (OR 7.004; 95\% CI 1.783-27.505; $p=0.005$ ), Ln (proteinuria) (OR 5.939; 95\% CI 1.055-33.436; $p=0.043$ ), Ln (sPLA2R-Ab) (OR 2.150; 95\% CI 1.293-3.577; $p=0.003$ ), and $\mathrm{Ln}(\mathrm{U} \alpha 1 \mathrm{~m} / \mathrm{Cr})(\mathrm{OR} 2.808 ; 95 \%$ CI 1.035-7.619; $p=0.043)$ were each independently associated with outcome.

\section{Nomogram and model performance}

We created a nomogram to predict the progression of patients with pMN who presented with NS (Fig. 1). The nomogram was based on the following four independent prognostic factors: age $\geq 65$ years, levels of $\operatorname{Ln}$ (proteinuria), levels of $\mathrm{Ln}$ (sPLA2R-Ab), and levels of $\mathrm{Ln}(\mathrm{U} \alpha 1 \mathrm{~m} / \mathrm{Cr})$. A higher total score based on the sum of the assigned number of points for each factor in the nomogram was associated with a worse prognosis. The resulting model was internally validated using the bootstrap validation method. The nomogram demonstrated good levels of accuracy for estimating the risk of progression, with an unadjusted C-index of 0.888 (0.814-0.940) and a bootstrap-corrected C-index of 0.869 . 
Table 1 Baseline clinical characteristics and outcomes of the pMN patients investigated in this study

\begin{tabular}{|c|c|}
\hline Parameters & $n(\%)$ or median $(\mathrm{IQR})(n=111)$ \\
\hline Sex, male/female & $68(61.3) / 43(38.7)$ \\
\hline Age, years & $57(41-66)$ \\
\hline Diabetes, $n(\%)$ & $13(11.7)$ \\
\hline Hypertension, $n(\%)$ & $55(49.5)$ \\
\hline Systolic blood pressure, $\mathrm{mmHg}$ & $132.0(123.0-140.0)$ \\
\hline Diastolic blood pressure, $\mathrm{mmHg}$ & $80.0(720-85.0)$ \\
\hline Albumin, $\mathrm{g} / \mathrm{L}$ & $26.0(23.0-29.0)$ \\
\hline Serum creatinine, $\mu \mathrm{mol} / \mathrm{L}$ & $67.0(56.0-80.0)$ \\
\hline eGFR, $\mathrm{mL} / \mathrm{min} / 1.73 \mathrm{~m}^{2}$ & $99.2(85.9-114.6)$ \\
\hline \multicolumn{2}{|l|}{ CKD stage, $n(\%)$} \\
\hline 1 & $77(69.4)$ \\
\hline 2 & $28(25.2)$ \\
\hline 3 & $6(5.4)$ \\
\hline sPLA2R-Ab positive, $n(\%)$ & $81(73.0)$ \\
\hline Levels of sPLA2R-Ab, RU/mL & $85.9(10.8-349.0)$ \\
\hline Hemoglobin, g/L & $132.0(119.0-144.0)$ \\
\hline Serum IgG, mg/dL & $601(433.5-738.0)$ \\
\hline Total cholesterol, mmol/L & $7.2(6.1-8.5)$ \\
\hline Triglyceride, $\mathrm{mmol} / \mathrm{L}$ & $2.3(1.5-3.2)$ \\
\hline $\mathrm{HDL}-\mathrm{C}, \mathrm{mmol} / \mathrm{L}$ & $1.2(1.1-1.6)$ \\
\hline LDL-C, mmol/L & $4.5(3.8-5.7)$ \\
\hline Proteinuria, $\mathrm{g} / 24 \mathrm{~h}$ & $5.7(4.5-7.2)$ \\
\hline Urinary $\mathrm{IgG} / \mathrm{Cr}, \mathrm{mg} / \mathrm{g}$ & $14.7(8.7-29.1)$ \\
\hline Urinary Tf, mg/g & $2.2(1.3-4.0)$ \\
\hline Urinary $\alpha 1 \mathrm{~m} / \mathrm{Cr}, \mathrm{mg} / \mathrm{mg}$ & $307.5(207.7-469.9)$ \\
\hline Urinary NAG/Cr, U/g & $38.6 \pm 18.6$ \\
\hline \multicolumn{2}{|l|}{ Kidney pathology, $n(\%)$} \\
\hline $\mathrm{IF} \operatorname{IgG}(+)$ & $111(100)$ \\
\hline $\operatorname{IF} \operatorname{IgA}(+)$ & $18(16.2)$ \\
\hline $\operatorname{IF} \operatorname{IgM}(+)$ & $32(28.8)$ \\
\hline $\mathrm{IF} \mathrm{C3}(+)$ & $93(83.8)$ \\
\hline $\operatorname{IF~C1q~(+)~}$ & $25(22.5)$ \\
\hline \multicolumn{2}{|l|}{ MN stage, $n(\%)$} \\
\hline I & $51(45.9)$ \\
\hline II & $45(40.5)$ \\
\hline III & $6(5.4)$ \\
\hline Obsolescent glomeruli, \% & $2.1(0-6.7)$ \\
\hline Focal segmental glomerular sclerosis, $n(\%)$ & $20(18.0)$ \\
\hline Acute tubular injury, $n(\%)$ & $23(20.7)$ \\
\hline \multicolumn{2}{|l|}{ Tubular atrophy } \\
\hline Stage 0 & $93(83.8)$ \\
\hline Stage 1 & $18(16.2)$ \\
\hline \multicolumn{2}{|l|}{ Interstitial fibrosis } \\
\hline Stage 0 & $92(82.9)$ \\
\hline Stage 1 & $19(17.1)$ \\
\hline Vascular hyalinosis, $n(\%)$ & $54(48.65)$ \\
\hline Immunosuppressive therapy, $n(\%)$ & $79(71.2)$ \\
\hline Complete remission, $n(\%)$ & $59(53.2)$ \\
\hline Partial remission, $n(\%)$ & $31(27.9)$ \\
\hline Progression, $n(\%)$ & $21(18.9)$ \\
\hline ESRD, $n(\%)$ & $11(9.9)$ \\
\hline Follow-up time, months & $40.0(25.0-58.0)$ \\
\hline
\end{tabular}

$\overline{C K D}$ chronic kidney disease, eGFR estimated glomerular filtration rate, ESRD end-stage renal disease, $H D L-C$ high-density lipoprotein cholesterol, $I F$ immunofluorescence $I Q R$ interquartile range, $L D L-C$ lowdensity lipoprotein cholesterol, $\alpha 1 m \alpha 1$-microglobulin, $M N$ membranous nephropathy, $N A G N$-acetyl- $\beta$-Dglucosaminidase, $s P L A 2 R-A b$ serum phospholipase A2 receptor antibody, $T f$ transferrin 
The calibration plots revealed good levels of agreement for the prediction of progression and risk estimation, as confirmed by the nomogram (Fig. 2).

In addition, an ROC curve was generated to compare the prognostic values of the identified risk factors with the nomogram scores. The nomogram score [area under the curve (AUC) $0.888,95 \%$ CI $0.814-0.940$ ) was better than each of the three risk factors alone, including Ln_sPLA2R-Ab (AUC 0.769, 95\% CI 0.679-0.843), Ln_proteinuria (AUC 0.653;

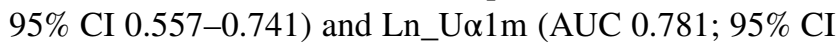
0.692-0.854). This suggested that the risk score had better levels of discrimination for predicting the progression of patients with pMN who presented with NS (Fig. 3).

\section{Risk of progression based on nomogram scores}

The nomogram score for an individual patient was defined as the weighted sum of the individual predictors, with weights equal to the regression coefficients in the final model; scores were calculated as follows: nomogram score $=32 \times(1$ if age $\geq 65$ years $)+29.4 \times[\operatorname{Ln}$ (proteinuria) -1.2$]+12.5 \times \operatorname{Ln}$ $(\mathrm{sPLA} 2 \mathrm{R}-\mathrm{Ab})+16.9 \times[\mathrm{Ln}(\mathrm{U \alpha} 1 \mathrm{~m} / \mathrm{Cr})-4.0]$. According to the Youden index, the optimal cutoff value for the nomogram score when predicting progression was 117.8. The sensitivity, specificity, positive predictive value, and negative predictive value were $95.2 \%, 72.2 \%, 44.4 \%$, and $98.5 \%$, respectively, when considered across the entire cohort (Table 3). Based on the optimal prognostic cutoff value, we categorized all patients into two risk groups: low-risk $(n=66 ;$ score $\geq 117.8)$ and high-risk $(n=45$; score $<177.8)$. We found that 20 of the 45 patients in the high-risk group showed progression compared to only 1 of the 66 patients in the low-risk group at the end of follow-up (Chi-squared Fisher's test $p<0.001$ ) (Table 4).

\section{Discussion}

Nomograms are increasingly used by clinicians to predict disease and involve simple-to-use digital interfaces that increase predictive power by integrating multiple independent predictors. In the current study, we created a nomogram that includes four independent risk factors (older age, sPLA2R-Ab, proteinuria, and urinary $\alpha 1 \mathrm{~m}$ ) to predict the progression of patients with pMN who present with NS. This model showed a good level of discrimination and had an excellent $\mathrm{C}$-index of 0.888 . Furthermore, the model was validated by bootstrap resampling. In addition, this novel nomogram provided us with a formula to calculate an individual's risk score with an optimal cutoff value of 117.8 . This may help clinicians to individualize treatment plans and follow-up strategies. To the best of our knowledge, this is the first available nomogram based on baseline parameters for predicting the outcome of patients with $\mathrm{pMN}$ who present with NS.

In our study, we found that older age, sPLA2R-Ab, proteinuria, and urinary $\alpha 1 \mathrm{~m}$ were significant independent predictors of renal outcome in $\mathrm{pMN}$ patients who present with NS. The association between age and prognosis in pMN patients was analyzed in previous studies. For example, Kim et al. [19] studied 135 Korean patients with pMN and found that older age ( $>60$ years) was a significant predictor for a lower rate of complete remission and also showed a higher rate of progression to ESRD although the treatment modalities were similar to those of younger patients. A study from China also demonstrated that age was an independent predictor for a combined outcome consisting of renal function progression, ESRD, and death [20]. These findings are consistent with the present results and indicate that older patients with $\mathrm{pMN}$ have an unfavorable clinical course and require more active treatment strategies. However, older age has also been associated with the occurrence of infectious complications in patients who had received immunosuppressants [21]. Collectively, these results suggest that treatment strategy should be individualized in older patients to balance both risks and benefits.

We also confirmed a significant association between baseline sPLA2R-Ab levels and renal outcome in $\mathrm{pMN}$ patients who present with NS. These findings are consistent with previous studies [22-25]. However, the precise threshold for the predictive value of sPLA2R-Ab remains controversial. In the extended Evaluate Rituximab Treatment for Idiopathic Membranous Nephropathy (GEMRITUX) study, investigators suggested that a baseline PLA2R-Ab level $<275$ RU/ $\mathrm{mL}$ was independently associated with complete or partial remission of proteinuria at 6 months [10]. In another study involving pMN patients after rituximab treatment, the probability of achieving clinical remission progressively decreased from the lowest tertile (14-86 RU/mL) to the middle tertile (87-204 RU/mL) and the highest tertile (> $204 \mathrm{RU} / \mathrm{mL}$ ) [26]. The differences between these studies may be attributed to the method of detection, ethnicity, baseline renal function, the ratio of patients with nephrotic-range proteinuria, treatment strategies, and the outcome definition. Therefore, considering the wide distribution of sPLA2R-Ab levels in clinical practice, sPLA2R-Ab was recognized as a continuous variable in our predictive nomogram and could provide more accurate information.

A previous study reported that persistent high-grade proteinuria ( $>6$ months of proteinuria $>8 \mathrm{~g} /$ day) was associated with a high risk of progressive loss of kidney function in patients with membranous nephropathy according to the 
Table 2 Logistic regression model showing the association of different variables with progression

\begin{tabular}{|c|c|c|c|c|}
\hline \multirow[t]{2}{*}{ Variable } & \multicolumn{2}{|l|}{ Univariable analysis } & \multicolumn{2}{|l|}{ Multivariable analysis } \\
\hline & OR $(95 \% \mathrm{CI})$ & $p$ value & OR $(95 \% \mathrm{CI})$ & $p$ value \\
\hline \multicolumn{5}{|l|}{ Factors selected } \\
\hline \multicolumn{5}{|l|}{ Age, years } \\
\hline$<65$ & 1 (reference) & & 1 (reference) & \\
\hline$\geq 65$ & $4.724(1.727-12.917)$ & $0.002 *$ & $7.004(1.783-27.505)$ & $0.005^{*}$ \\
\hline Ln (proteinuria, g/24 h) & 4.408 (1.093-17.774) & $0.037 *$ & $5.939(1.055-33.436)$ & $0.043 *$ \\
\hline Ln (sPLA2R-Ab, U/mL) & $1.002(1.001-1.004)$ & $0.001 *$ & $2.150(1.293-3.577)$ & $0.003^{*}$ \\
\hline $\mathrm{Ln}(\mathrm{U} \alpha 1 \mathrm{~m} / \mathrm{Cr}, \mathrm{mg} / \mathrm{mg})$ & $1.002(1.001-1.004)$ & $0.001 *$ & $2.808(1.035-7.619)$ & $0.043^{*}$ \\
\hline \multicolumn{5}{|l|}{ Factors not selected } \\
\hline \multicolumn{5}{|l|}{ Sex } \\
\hline Male & 1 (reference) & & & \\
\hline Female & $1.745(0.620-4.917)$ & 0.292 & & \\
\hline \multicolumn{5}{|l|}{ Hypertension } \\
\hline Without & 1 (reference) & & & \\
\hline With & $1.150(0.444-2.976)$ & 0.773 & & \\
\hline \multicolumn{5}{|l|}{ Diabetes } \\
\hline Without & 1 (reference) & & & \\
\hline With & $2.118(0.584-0.768)$ & 0.254 & & \\
\hline Albumin, g/L & $0.826(0.729-0.937)$ & $0.003 *$ & & \\
\hline Serum creatinine, $\mu \mathrm{mol} / \mathrm{L}$ & $1.015(0.996-1.034)$ & 0.131 & & \\
\hline Serum $\operatorname{IgG}, \mathrm{mg} / \mathrm{dL}$ & $0.999(0.996-1.001)$ & 0.232 & & \\
\hline \multicolumn{5}{|l|}{ CKD stage } \\
\hline CKD 1 & 1.0 (reference) & & & \\
\hline CKD 2/3 & $4.121(1.533-11.080)$ & $0.005^{*}$ & & \\
\hline $\operatorname{Ln}(\mathrm{UIgG} / \mathrm{Cr}, \mathrm{mg} / \mathrm{g})$ & $1.022(1.002-1.043)$ & $0.032 *$ & & \\
\hline Ln $(\mathrm{UTf} / \mathrm{Cr}, \mathrm{mg} / \mathrm{g})$ & $2.850(1.358-5.983)$ & $0.006^{*}$ & & \\
\hline Ln (NAG/Cr, U/g) & $1.041(1.014-1.068)$ & $0.002 *$ & & \\
\hline \multicolumn{5}{|l|}{ MN stage } \\
\hline Stage I & 1.0 (reference) & & & \\
\hline Stage II/III & $1.167(0.447-3.042)$ & 0.753 & & \\
\hline \multicolumn{5}{|l|}{ Acute tubular injury } \\
\hline Without & 1.0 (reference) & & & \\
\hline With & $1.250(0.404-3.867)$ & 0.699 & & \\
\hline \multicolumn{5}{|c|}{ Focal segmental glomerular sclerosis } \\
\hline Without & 1.0 (reference) & & & \\
\hline With & $1.562(0.496-4.920)$ & 0.446 & & \\
\hline \multicolumn{5}{|l|}{ Tubular atrophy } \\
\hline Stage 0 & 1.0 (reference) & & & \\
\hline Stage 1 & $1.851(0.578-5.925)$ & 0.300 & & \\
\hline \multicolumn{5}{|l|}{ Interstitial fibrosis } \\
\hline Stage 0 & 1.0 (reference) & & & \\
\hline Stage 1 & $1.696(0.535-5.383)$ & 0.370 & & \\
\hline \multicolumn{5}{|l|}{ Vascular hyalinosis } \\
\hline Without & 1.0 (reference) & & & \\
\hline With & $1.202(0.465-3.112)$ & 0.704 & & \\
\hline \multicolumn{5}{|l|}{ C3 deposits } \\
\hline Without & 1.0 (reference) & & & \\
\hline With & $2.054(0.434-9.716)$ & 0.364 & & \\
\hline
\end{tabular}

$C 3$ complement 3, $C K D$ chronic kidney disease, $L n$ natural logarithm, $M N$ membranous nephropathy, $N A G$ $N$-acetyl- $\beta$-D-glucosaminidase, $O R$ odds ratio, $s P L A 2 R-A b$ serum phospholipase A2 receptor antibody, Ualm urinary $\alpha 1$-microglobulin, UTf urinary transferrin

$* p<0.05$ 

Points
age

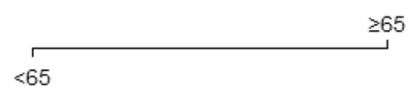
Ln_proteinuria

$\begin{array}{lllllllll}1.2 & 1.4 & 1.6 & 1.8 & 2 & 2.2 & 2.4 & 2.6 & 2.8\end{array}$
Ln_sPLA2R_Ab
0
1
65

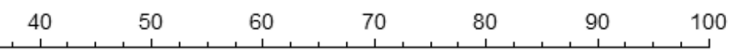
Ln_Ua1m

\begin{tabular}{llllllll}
\hline 4 & 1 & 1 & 1 & 1 & 1 & 1 \\
& 4.5 & 5 & 5.5 & 6 & 6.5 & 7 & 7.5
\end{tabular}
Total Points

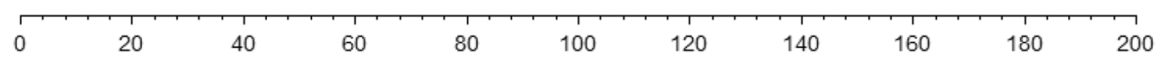
Probability of progression

\begin{tabular}{lllllllllllll}
\hline 0.001 & 0.01 & 0.05 & 0.1 & 0.2 & 0.3 & 0.4 & 0.5 & 0.6 & 0.7 & 0.8 & 0.9 & 0.95
\end{tabular}

Fig. 1 Nomogram predicting progression in pMN patients who present with NS. This nomogram was based on four independent prognostic factors: age $\geq 65$ years, $\mathrm{Ln}$ (proteinuria), $\mathrm{Ln}(\mathrm{sPLA} 2 \mathrm{R}-\mathrm{Ab})$ and $\mathrm{Ln}(\mathrm{U} \alpha 1 \mathrm{~m} / \mathrm{Cr})$

2019 KDIGO Controversies Conference Report [18]. However, risk stratification of the degree of proteinuria alone lacks specificity; measures of urinary protein composition may be a better method. In the present study, we measured levels of urinary $\mathrm{IgG}$, transferrin, and $\alpha 1 \mathrm{~m}$ corrected by creatinine in a single urine sample, thus representing high-, medium- and LMW protein, respectively. Univariable analysis further showed that all of these urinary biomarkers were associated with progression in pMN patients who present with NS. Nevertheless, multivariable analysis indicated that only $\alpha 1 \mathrm{~m}$ was independently associated with renal outcome. Similar to $\beta 2$-microglobulin, $\alpha 1 \mathrm{~m}$ is also a LMW protein and a known marker of tubulointerstitial injury. The results from two separate studies demonstrated that urinary $\alpha 1 \mathrm{~m}$ could replace $\beta 2$-microglobulin in the prediction of renal failure for pMN patients [13, 27]. Branten et al. [13] observed that urinary $\operatorname{IgG}$ was a useful marker for the severity of glomerular damage, and that urinary IgG excretion $\geq 250 \mathrm{mg} / 24 \mathrm{~h}$ had a robust relationship with renal survival in patients with pMN. However, our data were not able to validate the independent correlation between urinary IgG and renal outcome.
The reason for this discrepancy may be due to potential linear relationships between different predictive parameters.

In a recent study, Stangou et al. [28] investigated a large cohort of 752 patients with pMN over a long-term followup period (112-376 months). These authors found that the presence of FSGS and the degree of TA were significant independent parameters for the prediction of renal function outcome, as determined by multiple regression analysis. However, we did not identify any histological renal parameters that could independently predict the outcome of patients with pMN who present with NS. The differences in these results may be partly due to the relatively lower presence of FSGS and a milder degree of tubulointerstitial injury in our current patients. This also emphasizes the significance of monitoring early measurements of sPLA2R-Ab and urinary biomarkers, as these may be more important than histological changes when predicting outcome. In addition, ATI was observed in $20.7 \%$ (23/111) patients; we failed to identify any correlation between these histological changes and renal outcome in our cohort or any other studies. These findings suggest that the long-term renal outcome of $\mathrm{pMN}$ is more 
Fig. 2 Calibration plot comparing predicted and actual progression probabilities. This shows good agreement with regards to the prediction of progression between the risk estimation by nomogram confirmation

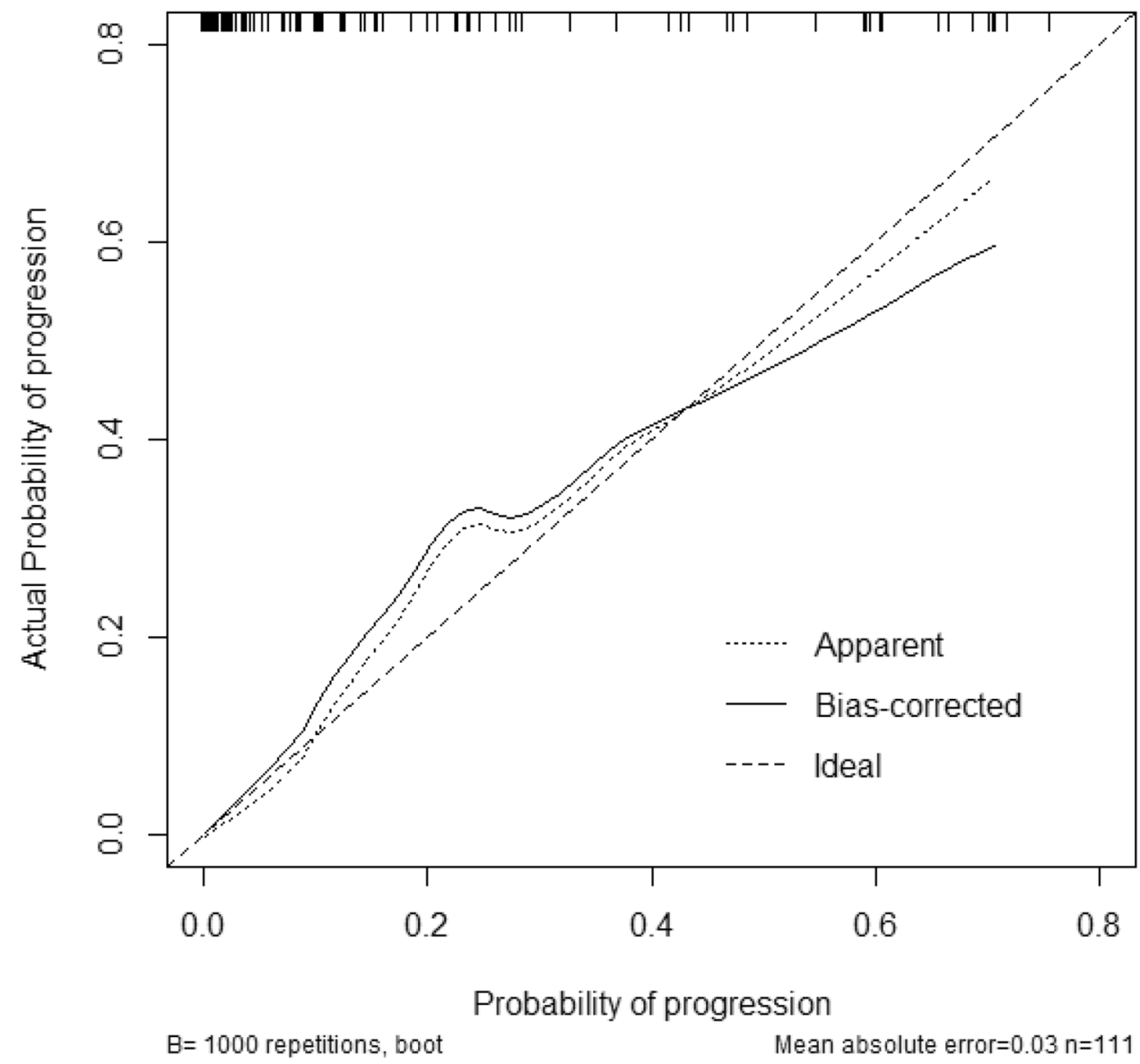

related to the presence and extent of chronic tubulointerstitial injury rather than ATI or glomerular pathology.

To date, we have had very limited options with regard to a predictive model that is capable of integrating multiple pMN risk factors. Cattran et al. [17] proposed a predictive model for renal prognosis in pMN patients based on dynamic changes of proteinuria and $\mathrm{CCr}$. Another group from China proposed a risk score based on baseline age, proteinuria, and eGFR to predict adverse outcomes in patients with pMN [20]. In the present study, we found that no serum or urinary biomarkers (e.g., sPLA2R-Ab, urinary IgG, or urinary $\alpha 1 \mathrm{~m}$ ) contributed to the risk score calculation. Our model has several strengths that need to be taken into consideration. First, the two existing models described above were both developed in pMN patients with more extensive clinical manifestations, including both nephrotic- and subnephrotic-range proteinuria, while our study focused on pMN patients with NS who need more attention with regard to treatment selection and prognostication. Second, we identified two important biomarkers (sPLA2R-Ab and Ua1m) as vital parameters that were able to provide early and accurate prediction of renal outcome. Third, we were able to rule out the confounding effects of immunosuppressive treatment on renal outcome because nearly almost all patients showing progression had received immunosuppressive treatment.

Our study also has some limitations that need to be considered. First, the patient cohort was recruited from a single center, and the follow-up period was relatively short. Second, the retrospective design could not exclude all confounding factors. Third, the model lacks external validation. Therefore, further studies with larger sample sizes and longer follow-up times are now needed to validate our predictive model and draw further conclusions.

\section{Conclusion}

We developed an optimized nomogram to provide accurate and early prognosis data for $\mathrm{pMN}$ patients with NS, a specific cohort of patients that require more clinical attention with regards to their treatment strategies and prognosis. Using this model, it is possible to determine the risk of a worse outcome for an individual patient. This can help clinicians to individualize treatment and improve the outcome of pMN. 
Fig. 3 Comparison of ROC curves for progression showing area under the curve (AUC) for the nomogram score $(0.888$; 95\% CI 0.814-0.940), Ln (sPLA2R-Ab) (AUC: 0.769; 95\% CI 0.679-0.843), Ln (proteinuria) $(0.653 ; 95 \% \mathrm{CI}$ $0.557-0.741), \operatorname{Ln}(\mathrm{U} \alpha 1 \mathrm{~m})$ (0.781; 95\% CI 0.692-0.854)

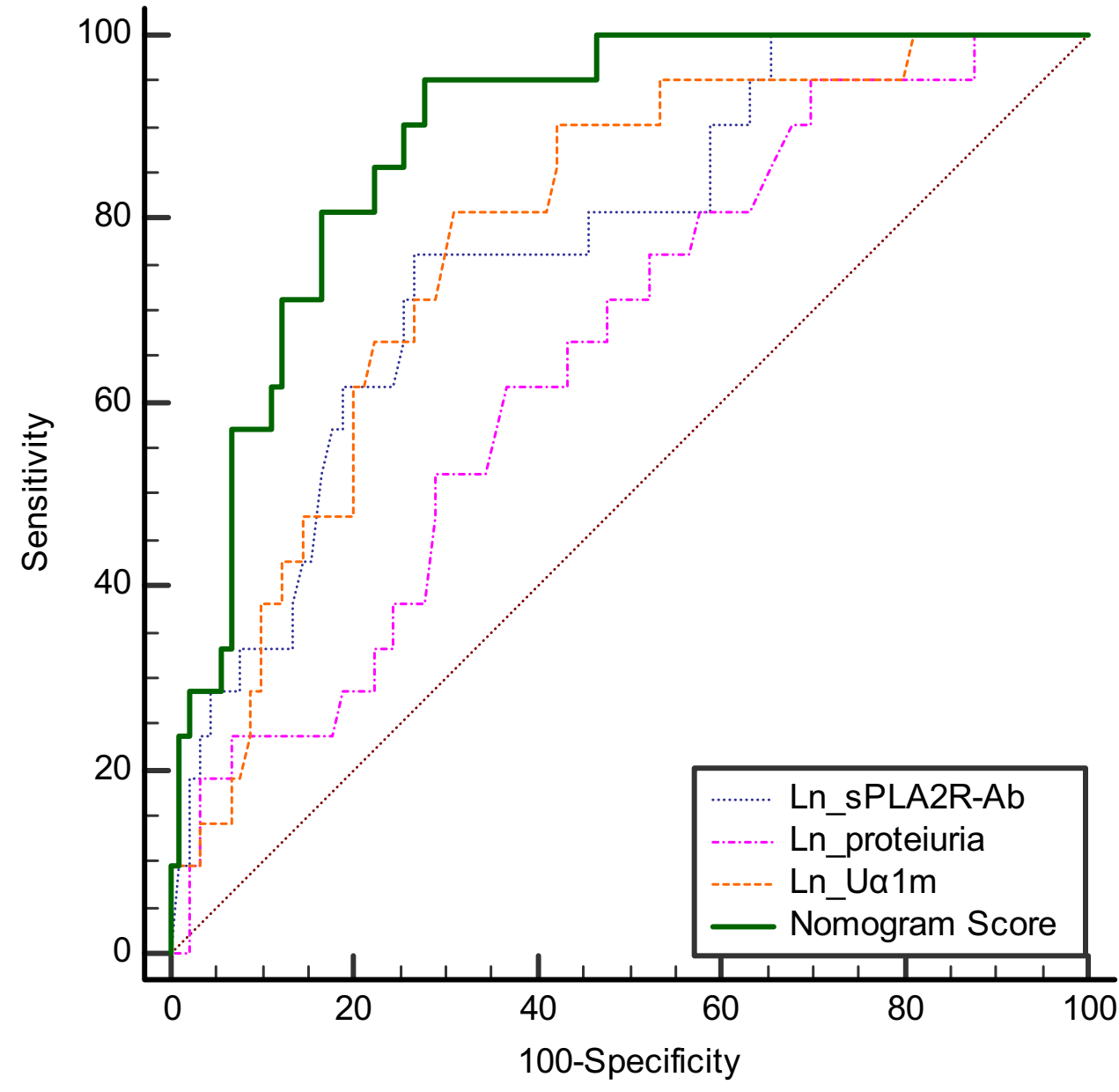

Table 3 Accuracy of the total nomogram score for predicting progression in pMN patients who presented with NS

\begin{tabular}{ll}
\hline Variable & Value (95\% CI) \\
\hline C-index & $0.888(0.814-0.940)$ \\
Cutoff score & 117.8 \\
Sensitivity, \% & $95.2(76.2-99.9)$ \\
Specificity, \% & $72.2(61.8-81.1)$ \\
Positive predictive value, \% & $44.4(36.1-53.1)$ \\
Negative predictive value, \% & $98.5(90.5-99.8)$ \\
Positive likelihood ratio & $3.43(2.4-4.8)$ \\
Negative likelihood ratio & $0.066(0.010-0.400)$ \\
\hline
\end{tabular}

Table 4 Association between the risk stratification of the nomogram score and outcome in $\mathrm{pMN}$ patients who presented with NS

\begin{tabular}{llll}
\hline & $\begin{array}{l}\text { Low-risk } \\
\text { group } \\
(n=66)\end{array}$ & $\begin{array}{l}\text { High-risk } \\
\text { group } \\
(n=45)\end{array}$ & $\begin{array}{l}p \text { value } \\
\text { Fisher's test }\end{array}$ \\
\hline Without progression, $n(\%)$ & $65(98.5)$ & $25(55.6)$ & $<0.001$ \\
With progression, $n(\%)$ & $1(1.5)$ & $20(44.4)$ & \\
\hline
\end{tabular}

Supplementary Information The online version contains supplementary material available at https://doi.org/10.1007/s11255-021-02859-x.

Acknowledgements We thank Dr. Xianguang Chen for his valuable advice.

Author contributions LL designed the experiments, performed the experiments, collected the data, performed the formal analysis, and wrote the manuscript. HW, BZ, XL, and YS performed the experiments and collected the data. YM designed experiments and reviewed/edited the manuscript. All authors reviewed the results and approved the final version of the manuscript.

Funding This work was supported by a grant from Beijing Hospital Nova Project (BJ-2018-131) to Lili Liu, a grant from Beijing Hospital Clinical Research 121 Project (121-2016008) to Yonghui Mao, and a grant from the Special Funds for Clinical Medical Research of the Chinese Medical Association (20010030795).

Availability of data and materials All data that support the conclusions of this manuscript are included within the article. 


\section{Declarations}

Conflict of interest The results presented in this paper have not been published previously in whole or part, except in abstract format. The authors declare that they have no competing interests.

Ethics approval and consent to participate The study was performed according to the Declaration of Helsinki and approved by the ethics committee of Beijing Hospital (no: 2018BJYYEC-140-01). Written informed consent was obtained from all patients.

Consent for publication All the authors read and approved the final manuscript and agree to publish this paper.

Open Access This article is licensed under a Creative Commons Attribution 4.0 International License, which permits use, sharing, adaptation, distribution and reproduction in any medium or format, as long as you give appropriate credit to the original author(s) and the source, provide a link to the Creative Commons licence, and indicate if changes were made. The images or other third party material in this article are included in the article's Creative Commons licence, unless indicated otherwise in a credit line to the material. If material is not included in the article's Creative Commons licence and your intended use is not permitted by statutory regulation or exceeds the permitted use, you will need to obtain permission directly from the copyright holder. To view a copy of this licence, visit http://creativecommons.org/licenses/by/4.0/.

\section{References}

1. Zhu P, Zhou FD, Wang SX et al (2015) Increasing frequency of idiopathic membranous nephropathy in primary glomerular disease: a 10-year renal biopsy study from a single Chinese nephrology centre. Nephrology 20(8):560-566

2. Beck LH Jr, Salant DJ (2010) Membranous nephropathy: recent travels and new roads ahead. Kidney Int 77(9):765-770

3. Cattran DC, Brenchley PE (2017) Membranous nephropathy: integrating basic science into improved clinical management. Kidney Int 91(3):566-574

4. Beck LH, Bonegio RG, Lambeau G et al (2009) M-type phospholipase A2 receptor as target antigen in idiopathic membranous nephropathy. N Engl J Med 361(1):11-21

5. Du Y, Li J, He F et al (2014) The diagnosis accuracy of PLA2R$\mathrm{AB}$ in the diagnosis of idiopathic membranous nephropathy: a meta-analysis. PLoS ONE 9:e104936

6. Hu SL, Wang D, Gou WJ et al (2014) Diagnostic value of phospholipase A2 receptor in idiopathic membranous nephropathy: a systematic review and meta-analysis. J Nephrol 27(2):111-116

7. Dai H, Zhang H, He Y (2015) Diagnostic accuracy of PLA2R autoantibodies and glomerular staining for the differentiation of idiopathic and secondary membranous nephropathy: an updated meta-analysis. Sci Rep 5:8803

8. Couser WG (2017) Primary membranous nephropathy. Clin J Am Soc Nephrol 12(6):983-997

9. Radhakrishnan J, Cattran DC (2012) The KDIGO practice guideline on glomerulonephritis: reading between the (guide)linesapplication to the individual patient. Kidney Int 82(8):840-856

10. Dahan K, Debiec H, Plaisier E et al (2017) Rituximab for severe membranous nephropathy: a 6-month trial with extended followup. J Am Soc Nephrol 28(1):348-358

11. Reichert LJM, Koene RAP, Wetzels JFM (1995) Urinary excretion of 2-microglobulin predicts renal outcome in patients with idiopathic membranous nephropathy. J Am Soc Nephrol 6(6): $1666-1669$
12. Reichert LJM, Koene RAP, Wetzels JF (1997) Urinary IgG excretion as a prognostic factor in idiopathic membranous nephropathy. Clin Nephrol 48(2):79-84

13. Branten AJ, du Buf-Vereijken PW, Klasen IS et al (2005) Urinary excretion of $\beta 2$-microglobulin and IgG predict prognosis in idiopathic membranous nephropathy: a validation study. J Am Soc Nephrol 16(1):169-174

14. Bazzi C, Rizza V, Casellato D et al (2014) Fractional excretion of IgG in idiopathic membranous nephropathy with nephrotic syndrome: a predictive marker of risk and drug responsiveness. BMC Nephrol 15:74

15. Liang Y, Wan J, Chen Y et al (2019) Serum anti-phospholipase A2 receptor (PLA2R) antibody detected at diagnosis as a predictor for clinical remission in patients with primary membranous nephropathy: a meta-analysis. BMC Nephrol 20(1):360

16. Qu Z, Zhang MF, Cui Z et al (2018) Antibodies against M-type phospholipase A2 receptor may predict treatment response and outcome in membranous nephropathy. Am J Nephrol 48(6):438-446

17. Cattran DC, Pei Y, Greenwood CM et al (1997) Validation of a predictive model of idiopathic membranous nephropathy: its clinical and research implications. Kidney Int 51(3):901-907

18. Floege J, Barbour SJ, Cattran DC et al (2019) Management and treatment of glomerular diseases (part 1): conclusions from a Kidney Disease: Improving Global Outcomes (KDIGO) Controversies Conference. Kidney Int 95(2):268-280

19. Kim Y, Yoon HE, Chung BH et al (2019) Clinical outcomes and effects of treatment in older patients with idiopathic membranous nephropathy. Korean J Intern Med 34(5):1091-1099

20. Xiaofan H, Jing X, Chenni G et al (2019) New risk score for predicting progression of membranous nephropathy. J Transl Med 17(1):41

21. Yamaguchi M, Ando M, Yamamoto R et al (2014) Patient age and the prognosis of idiopathic membranous nephropathy. PLoS ONE 9(10): 110376

22. Hoxha E, Thiele I, Zahner G, Panzer U, Harendza S, Stahl RAK (2014) Phospholipase A2 receptor autoantibodies and clinical outcome in patients with primary membranous nephropathy. J Am Soc Nephrol 25(6):1357-1366

23. Li WY, Zhao YL (2019) Prognostic value of phospholipase A2 receptor in primary membranous nephropathy: a systematic review and meta-analysis. Int Urol Nephrol 51(9):1581-1596

24. Mahmud M, Pinnschmidt HO, Reinhard L et al (2019) Role of phospholipase A2 receptor 1 antibody level at diagnosis for longterm renal outcome in membranous nephropathy. PLoS ONE 14(9):e0221293

25. Wei SY, Wang YX, Li JS et al (2016) Serum anti-PLA 2 R antibody predicts treatment outcome in idiopathic membranous nephropathy. Am J Nephrol 43:129-140

26. Ruggenenti P, Debiec H, Ruggiero B et al (2015) Anti-phospholipase A2 receptor antibody titer predicts post-rituximab outcome of membranous nephropathy. J Am Soc Nephrol 26(10):2545-2558

27. Van den Brand JA, Hofstra JM, Wetzels JF (2012) Prognostic value of risk score and urinary markers in idiopathic membranous nephropathy. Clin J Am Soc Nephrol 7(8):1242-1248

28. Stangou MJ, Marinaki S, Papachristou E et al (2019) Histological grading in primary membranous nephropathy is essential for clinical management and predicts outcome of patients. Histopathology 75(5):660-671

Publisher's Note Springer Nature remains neutral with regard to jurisdictional claims in published maps and institutional affiliations. 